\title{
Chemical Abundances Variations and Kinematical Ages of Multiple Shell Planetary Nebulae and Their Haloes
}

\author{
M. A. Guerrero, A. Manchado
}

Instituto de Astrofísica de Canarias

We have carried out a comparative study of the chemical abundances and physical properties of the haloes and main nebulae on a sample of type I multiple shell PNe: M 1-46, NGC 2438, NGC 5882, NGC 6543, NGC 6720, NGC 6751, and NGC 6826.

The $\mathrm{N} / \mathrm{O}$ and $\mathrm{He} / \mathrm{H}$ ratios obtained are in agreement with the theoretical predictions that low- and intermediate-mass star models propose for progenitor stars initially as massive as $1-2.5 \mathrm{M}_{\odot}$. No clear abundances gradient exists between the haloes and the main nebulae, thus indicating that the chemical enrichment during the formation of the different shells is very small.

The suspected connection between thermal pulses and enhanced mass-loss phases, when the shell ejection occurs, has also been tested for the PNe in this sample. It has been found that there is a good agreement between the theoretical interpulse time-lap and the observed intershell time of the haloes of type I multiple shell PNe (Fig. 1). However, it should be noted that the intershell time shows a trend to have lower values than the predicted interpulse times.

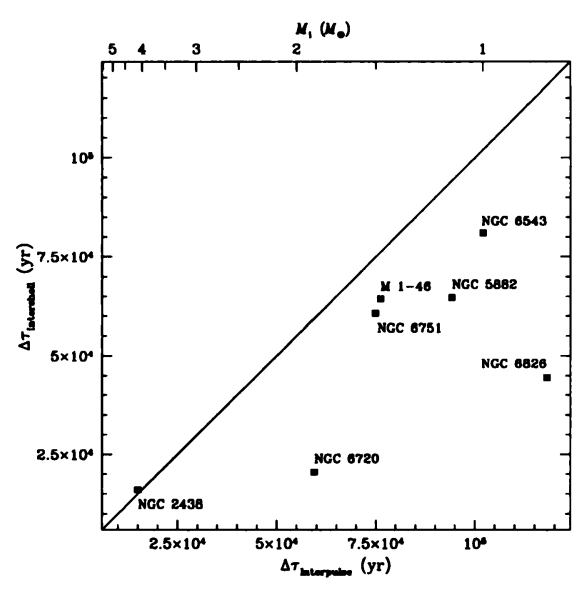

Figure 1. Intershell time $\Delta \tau_{\text {intershell }}$ versus theoretical time between thermal pulses

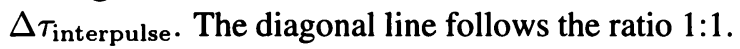

The spectra of the haloes are very different to these of the main nebulae, thus indicating that haloes are not due to light reflection. The ionization mechanism of haloes has also been investigated. The 'hardening' of the radiation indicates that it is due to stellar UV radiation which leaks through the material more recently ejected. 